\title{
Predator presence and recent climatic warming raise body temperatures of island lizards
}

\author{
Felix Landry Yuan ${ }^{1}$, Shun Ito $^{2}$, Toby Pak Nok Tsang ${ }^{3}$, Takeo Kuriyama ${ }^{4}$, Kaede \\ Yamazaki $^{5}$, Timothy Bonebrake ${ }^{3}$, and Masami Hasegawa ${ }^{6}$ \\ ${ }^{1}$ University of Hong Kong \\ ${ }^{2}$ Tohoku Gakuin University \\ ${ }^{3}$ The University of Hong Kong \\ ${ }^{4}$ University of Hyogo \\ ${ }^{5}$ Toho University - Narashino Campus \\ ${ }^{6}$ Toho University
}

September 28, 2020

\begin{abstract}
In ectothermic predator-prey relationships, the capacity for prey to successfully evade predation will depend upon physiological and behavioural responses that relate to both players' thermal biology. On the Izu Islands of Japan, we investigated how a prey lizard species has responded physiologically and thermally to the presence of a snake predator over evolutionary time in addition to recent climatic warming. Foraging lizard body temperatures have increased by $1.0^{\circ} \mathrm{C}$ from 1981 to 2019 while lizard body temperatures were $3.4^{\circ} \mathrm{C}$ warmer on islands where the snake predator is present relative to snake-free islands. We also found that warmer prey body temperatures result in faster running speeds of the prey at temperatures suboptimal for the snake predator. The results show that lizard body temperatures have increased with warming but not to the same extent as that exerted by predation pressure. However, further warming could irrevocably alter this and other ectothermic predator-prey relationships.
\end{abstract}

\section{Predator presence and recent climatic warming raise body temperatures of island lizards}

Félix Landry Yuan ${ }^{\mathrm{a} * 1}$, Shun Ito ${ }^{\mathrm{b} 1}$, Toby P. N. Tsang ${ }^{\mathrm{a}}$, Takeo Kuriyama ${ }^{\mathrm{c}}$, Kaede Yamazaki ${ }^{\mathrm{d}}$, Timothy C. Bonebrake $^{\mathrm{a}}$ and Masami Hasegawa ${ }^{\mathrm{d}}$

Short running title: Snakes and climate raise lizard temperatures

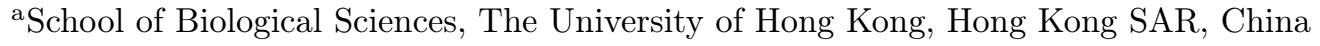

${ }^{\mathrm{b}}$ Graduate School of Life Science, Tohoku University, Aoba-ku, Sendai, Japan

${ }^{\mathrm{c}}$ Institute of Natural and Environmental Sciences, University of Hyogo, Tamba, Japan

${ }^{\mathrm{d}}$ Department of Biology, Faculty of Science, Toho University, Funabashi, Chiba, Japan

*Corresponding author. E-mail address: flyuan@connect.hku.hk. Telephone number: +852 5649 2201. Mailing address: School of Biological Sciences, Kadoorie Biological Sciences Building, The University of Hong Kong, Pok Fu Lam Road, Hong Kong SAR, China.

${ }^{1}$ These authors contributed equally to the manuscript. 
Statement of authorship: FLY, SI, MH and TCB designed the study. FLY, TK, KY, YK and MH collected the data, while FLY, TPNT and SI performed the analyses. FLY and SI wrote the manuscript, and all authors significantly contributed to revisions.

Data accessibility statement: Data supporting the results will be archived in Dryad should the manuscript be accepted.

Keywords: prey-predator, interspecific interactions, body temperature, thermal performance, ectotherm, climate change, japan, lizard, snake

Type of article: Letters

Words in abstract: 147

Words in main text: 4965

Number of references: 56

Number of figures: 5

Number of tables: 0

Number of text boxes: 0

\section{ABSTRACT}

In ectothermic predator-prey relationships, evasion of predation by prey depends on physiological and behavioural responses relating to both players' thermal biology. On Japan's Izu Islands, we investigated a prey lizard's physiological and thermal responses to the presence of a snake predator over evolutionary time in addition to recent climatic warming. Foraging lizard body temperatures have increased by $1.0^{\circ} \mathrm{C}$ from 1981 to 2019 overall, yet were $3.4^{\circ} \mathrm{C}$ warmer on snake islands relative to snake-free islands. We also detected snake predator-induced selection on hind leg length, which in turn is a major determinant for sprint speed only in lizard populations exposed to predation by snakes. Accordingly, we found that warmer prey body temperatures result in faster sprint speeds by the prey at temperatures suboptimal for the snake predator, and therefore contribute to escaping predation. Given recent climatic change, further warming could irrevocably alter this and other ectothermic predator-prey relationships.

\section{INTRODUCTION}

In ectotherms, where body temperatures and physiological processes are dependent on immediate thermal environments, the prey's survival under predation is tied to the accessibility of body temperatures to achieve their thermal optimum for escaping by rapid movement (Hertz et al. 1983; Chai and Srygley 1990; Cooper 2000). To successfully evade predation when chased, a prey's thermal optimum for escape, in addition to its locomotory speed at that temperature, should surpass that of the predator for chasing (Grigaltchik et al. 2012; Dell et al. 2014; Öhlund et al. 2015; Figueira et al. 2019). Ectothermic prey-predator dynamics become increasingly complex when we consider how recent climate change has and will foreseeably continue to impact both players (Grigaltchik et al. 2012; Laws 2017). In a warming world, changes in the available thermal landscapes can manifest at the order of microhabitats, which is especially challenging for lizards because of their dependency on thermoregulation to reach body temperatures adequate for behaviours supporting survival and reproduction (Sinervo et al. 2010). Considering the thermally dependent nature of predator evasion, the evolution of a prey species' thermal physiology and behaviour should be strongly affected by both their predators and changes in environmental temperatures.

Within the Galapagos Archipelago on Santa Fe Island, Christian and Tracy (1981) found that the success of predation by Galapagos hawks (Buteo galapagoensis ) on hatchling land iguanas (Conolophus pallidus ) is highest at times of the day when iguana body temperatures are suboptimal for sprinting. Conversely, at times when iguanas were capable of attaining body temperatures optimal for reaching maximal sprint speeds, predation success by the hawks was much lower. Similarly, on Isla Plaza Sur, Snell et al. (1988) found 
that lava lizards (Tropidurus albemarlensis ) run faster on the side of the island with less vegetation and greater exposure to predators. Oceanic island systems such as the Galapagos Islands are ideal for studying ecological processes shaping life history because they formed in isolation with no prior connection to other landmasses. Once colonized by limited overseas dispersal, the flora and fauna on each island develops along unique evolutionary trajectories shaping each island's present-day diversity (Whittaker et al. 2017). In the case where multiple populations of a single species remain isolated across an archipelago over evolutionary time, it allows for an untangling of the effects of different selective pressures (Kuriyama et al. 2011; Brandley et al. 2014).

Located off the southeastern coast of Japan's mainland, the volcanic Izu Islands formed independently less than a million years ago, rendering them completely vacant for colonization by species occupying adjacent land (Kaneoka et al. 1970). The low number of reptile species in the Izu Islands relative to tropical or other less-isolated regions (Hasegawa 2003) provides an opportunity to single out the selection pressures of prey-predator relationships on thermal physiology and behaviour. Notably, one endemic lizard species, Okada's five-lined skink (Plestiodon latiscutatus ), is dominant and distributed across all of the system's major islands, while one of its major predators, the Japanese four-lined rat snake (Elaphe quadrivirgata), is also found on most, but not all of these islands (Hasegawa and Moriguchi 1989), resulting in a number of insular skink populations free from snake predation. Therefore, taking into account the thermal dependency of both predator and prey in this system, we can expect adaptions in the thermal physiology and behaviour of $P$. latiscutatus in order to escape predation by E. quadrivirgata across space and time.

With our study spanning nearly 40 years, we investigated variations in the foraging body temperature of $P$. latiscutatus as a function of snake predator presence as well as climatic warming over the study period. We also follow the sequence through which field-active body temperatures matter in this prey-predator relationship by quantifying the physiological and morphological components of the thermal dependency of locomotion and its overall role in the evasion of the snake predator by the prey lizard.

\section{METHODS}

\section{Study site and species}

We collected data on seven Izu Islands, namely Hachijo-Kojima $\left(33.13^{\circ} \mathrm{N}, 139.69^{\circ} \mathrm{E}\right)$, Miyake $\left(34.08^{\circ} \mathrm{N}\right.$, $\left.139.52^{\circ} \mathrm{E}\right)$, Mikura $\left(33.88^{\circ} \mathrm{N}, 139.60^{\circ} \mathrm{E}\right)$, Kozu $\left(34.22^{\circ} \mathrm{N}, 139.15^{\circ} \mathrm{E}\right)$, Oshima $\left(34.74^{\circ} \mathrm{N}, 139.40^{\circ} \mathrm{E}\right)$, Niijima $\left(34.37^{\circ} \mathrm{N}, 139.26^{\circ} \mathrm{E}\right)$ and Toshima $\left(34.52^{\circ} \mathrm{N}, 139.28^{\circ} \mathrm{E}\right)$, ranging in size from approximately 6 to 9076 ha (Figure S1 in Supporting Information). These volcanic islands are located along an axis radiating southwards from the Izu peninsula of mainland Japan, and are generally subject to the same mild climate carried over by the Kuroshio current (Hasegawa 1994; Brandley et al. 2014), with average annual air temperatures and total precipitation ranging from 17.3 to $18.8{ }^{\circ} \mathrm{C}$, and 1810 to $2958 \mathrm{~mm}$, respectively, for the year 2018 (http://www.data.jma.go.jp/).

P. latiscutatus is the most abundant lizard species overall in this system and is found on all islands, where, with little interspecific competition, it is usually found in high abundance, especially on islands with low predation (Hasegawa 1994). Amongst its terrestrial predators, E. quadrivirgata is the most widespread and abundant, with Oshima being the only island where there are other native species preying on the lizard. These include snakes, such as Lycodon orientalis and Gloydius blomhoffii, as well as the Japanese weasel (Mustela itatsi), the only mammalian carnivore native to any of the islands (Hasegawa 1994). Therefore, aside from Oshima, the only other native predators to the lizards on the islands are birds (Hasegawa 1990, Hasegawa et al. 1996), notably the grey-faced buzzard (Butastur indicus ) and the Izu island thrush (Turdus celaenops ). Given that E. quadrivirgata is present on five of our study islands and absent on two, HachijoKojima and Miyake, the only potential predation pressure for lizards on the latter two islands at the time of data collection was from avian predators, whereas predation on Mikura, Kozu, Niijima and Toshima was mainly by E. quadrivirgata (Hasegawa 1994). The evolutionary history of the relationship between $P$. latiscutatusand E. quadrivirgata is outlined in Appendix 1 of the Supporting Information.

Foraging body temperature, sprint speed, morphology and fitness data collection 
We collected lizard foraging body temperature data in the field yearly from 1981 to 1991, and then again in 2004, 2005, 2018 and 2019, all between the months of April to October, and during the day (6:30 17:00), when lizards are active. When encountering a lizard, we first observed its behaviour and then caught it using a mealworm-baited (Tenebrio molitor ) fishing pole. We identified foraging behaviour in lizards actively searching their surroundings and flicking their tongues, with bodies not pressed against substrates as they would be during basking. Upon capture, we immediately measured body temperature by inserting a $\pm 0.1{ }^{\circ} \mathrm{C}$, K-type thermocouple (TX-10, Yokogawa, Japan) into the cloaca. We then measured the nearest air temperature in the shade, noted time of day, marked the individual with a marker, and then released it at the site of capture. All data are thus known to be from separate individuals. We should also note that our data for Miyake island lizards are limited to 1983 due to the incidental population collapse of P. latiscutatus following the deliberate release of the Japanese weasel (Mustela itatsi) for the control of rats in the mid 1980's (Hasegawa 2003). We retrieved observatory air temperature data from the Japan Meteorological Agency (http://www.data.jma.go.jp/) both as daily mean averages for each year, and at the finest time scale available to match that of our collected body temperature (the finest being 10 minute averages, and the coarsest being day averages). Such data however were not available for Kozu from 1981 to 1991, or for Mikura or Toshima at any time. Although data for Hachijo-Kojima were not available, we used the air temperature data for the larger, adjacent Hachijojima as a proxy, which lies $\sim 7 \mathrm{~km}$ away.

We collected sprint speed data for lizards in two different ways - directly on open field terrain, for a better comparison with snake crawling speeds, and on a racetrack, to compare lizard sprint speeds between two islands with and without the snake predator in a controlled setting. We measured racetrack sprint speed for lizards on Hachijo-Kojima and Kozu islands from May to June 2018 and from July to August 2019. For this, we brought a $90 \mathrm{~cm}$ long wooden racetrack marked at $5 \mathrm{~cm}$ intervals, as well as a camera and tripod, to the field. We then caught lizards using the baited fishing pole method and either briefly placed them in a bucket, or immediately coerced them to run along the track, with each individual only running once. The lizards sprinted along the track and into a cloth bag, at which point we immediately measured body temperature via the cloaca, as well as morphological measurements, which included snout-vent length (SVL), total tail length, tail break distance (length between the tail base and the first point at which the tail has broken off in the past) and mass. For the 2019 experiments, we additionally measured hind leg length (joint to tip of the longest toe) and hind foot length (bottom of the heel to tip of the longest toe). We video recorded each run at 60 frames per second to obtain a measurement of the fastest $10 \mathrm{~cm}$ run in $\mathrm{cm} / \mathrm{s}$.

We measured open field sprint speed for lizards on Kozu from July to September 1990, on concrete and earthen surfaces they regularly occupy. Within 2-3 $\mathrm{m}$ from a lizard, and with stopwatch in hand, one person would stomp their foot, prompting the lizard to run. Once the lizard reached a visible landmark approximately $1-3 \mathrm{~m}$ away, such as a rock or crack, we clocked the time, immediately caught the individual by hand or baited fishing pole, and inserted a K-type thermocouple into the cloaca to measure body temperature. We then measured the starting point to landmark distance to calculate sprint speed in $\mathrm{cm} / \mathrm{s}$. For all open field and racetrack sprint speed measurements, we conducted experiments on a variety of days and times of the year to include the range of temperatures lizards can experience in the field, and only included non-juvenile individuals (SVL, $>35 \mathrm{~mm}$ ). We also discounted sprint speed data obtained from individuals showing limb deformities or impairment.

We measured crawling speed for the snakes on Kozu in April 1994 and August 1996, as well as on Niijima in May 1996. For this, we caught individuals by hand in the field and briefly kept them in cloth bags to carry over to a flat earthen surface. We conducted experiments on different days and times of the year to include as much as possible the range of body temperatures snakes naturally experience in the field. With stopwatch in hand, we then released each snake with the head positioned at one marked spot on the ground and re-caught it after it had dashed a distance of approximately $2-3 \mathrm{~m}$, at which point we marked the exact position of the head at recapture, and immediately measured body temperatures via the cloaca by inserting a K-type thermocouple. We then measured the distance between the two marked positions, and together with the time clocked by the stopwatch, obtained an estimate of crawling speed in $\mathrm{cm} / \mathrm{s}$. For both lizard and snake experiments, we released all individuals at respective sites of capture following completion. 
To determine fitness in the context of survival against predation by snakes, we collected lizard morphological data including SVL, hind leg length, and tail break distance, from two islands with the snake predator (Kozu and Mikura) and two without (Hachijo-Kojima and Miyake), separately from sprint speed and body temperature measurements. We measured live individuals on Mikura in 1981, as well as on Kozu and HachijoKojima in 2019.

We measured SVL and tail break distance on Miyake individuals in 1979, before the weasel-induced population collapse, and fixed and preserved individuals in 10\% formalin and $70 \%$ ethanol, respectively. In 2020 , we measured the hind leg length of those preserved individuals. We estimated hind leg lengths when alive by major axis regression, with hind leg length when alive as the response and that when preserved as the predictor. We based the model on living and preserved individuals from Oshima $(\mathrm{n}=6)$, Mikura $(\mathrm{n}=14)$ and Kozu $(\mathrm{n}=22)$, for which we had measured hind leg length of the same individuals under both conditions $(y$ $\left.=9.48+0.80 x ; R^{2}=0.73\right)$.

\section{Statistical analyses}

We tested for differences in lizard foraging body temperatures between snake and snake-free islands as well as between the two periods of 1981-1991 and 2018-2019 by building a linear mixed-effects model using the "lme4" package (De Boeck et al. 2011) in R (R Core Team 2019), with air temperature in the shade as a covariate, snake presence and period as fixed effects, and island, month and year as random intercepts. We used the "MuMIn" package (Bartoń 2020) to calculate $\mathrm{R}^{2}$ values for the model.

To compare the thermal dependency of maximal locomotory speeds between Hachijo-Kojima and Kozu P. latiscutatus on the racetrack, and between E. quadrivirgata and Kozu P. latiscutatusin open field, we modelled thermal performance curves (Angilletta 2009). For this, we fit raw data for each group to four different non-linear models (Tables S1 and S2 in Supporting Information; Angilletta 2006). We then chose the best fitting model overall in each of the two comparisons based on yielded Akaike's information criterion (AIC), assuming a difference larger than 2 to be significant (Burnham and Anderson 2002), relative AIC weights $\left(w_{i}\right)$, residual sums of squares (RSS), as well as the biological relevance of yielded curve shapes, where an expected shape would see a gradual increase in performance with body temperature up until an optimum after which performance drops rapidly to zero (Angilletta 2006; Angilletta 2009). From the thermal performance curves, we extracted values pertinent to the characterization of the thermal dependency of locomotion (Logan et al. 2013). These included the thermal optimum for sprinting $\left(T_{\text {opt }}\right)$, maximal performance $\left(P_{\max }\right)$, and the range of body temperatures at which $95 \%$ of $P_{\max }$ is attainable. The latter estimate is termed the $B_{95}$ performance breadth, and is interpreted as the thermal optimum range (Hertz et al. 1983).

In addition to thermal physiology, we aimed to determine if lizards from Kozu, where the snake predator is present, also show adaptations in limb morphology relative to those from Hachijo-Kojima. This is a relevant morphological dimension to compare considering that lizards that have evolved longer hindlimbs run faster at both population and species level (Bonine and Garland 1999; Aerts et al. 2000). We thus tested for differences in hind leg and hind foot lengths, both as ratios of SVL, between lizards from the two islands with two-sampled t-tests. We then applied a Bayesian model to determine relationships between morphological traits and maximum sprint speeds for both lizard populations while accounting for multicollinearity in morphological traits. Here we used individual maximum sprint speed measurements rather than values extracted from modelled thermal performance curves. To remove the effect of temperature on sprint speed, we estimated relative sprint speed by regressing log-transformed body temperature against log-transformed sprint speed (Lleonart et al. 2000), where we used the standard major axis regression as the regression. We subsequently used the obtained residuals as estimates of relative sprint speed. We conducted the analysis using the "smart" package in R (Warton et al. 2012; R Core Team 2019). We constructed a hierarchical model consisting of four equations to estimate the contribution of each measurement to relative 
sprint speed (Zipkin et al. 2020; Figure S2). We defined the core of this model with the following equation:

$$
\begin{gathered}
\operatorname{rSP} P_{i} \sim \operatorname{Normal}\left(\beta_{1, k} \bullet \mathrm{HL}_{i}+\beta_{2, k} \bullet \mathrm{HF}_{i}+\beta_{3, k} \bullet \operatorname{Tail}_{i}+\beta_{4, k} \bullet \mathrm{SVL}_{i}+e p_{1, k}, \quad \sigma_{1}^{2}\right) \#(1) \\
i=1,2, \ldots N, k=1,2
\end{gathered}
$$

where $\operatorname{rS} P_{i}$ represents the value of the $i$ th relative sprint speed, $H L_{i}$ represents the value of $i$ th hind leg length, $H F_{i}$ represents the value of $i$ th hind foot length,Tail $l_{i}$ represents the value of $i$ th tail length,SV $L_{i}$ represent the value of $i$ th SVL, $\beta_{1, k}, \beta_{2, k}, \ldots, \beta_{4, k}$ are the coefficients of each variable, $e p_{1, k}$ is the fixed effect on the $k$ th population, $\sigma_{1}^{2}$ is the variance, $N$ is the number of individuals, $k$ is the index for the island (Hachijo-Kojima or Kozu). This model assumes that the contribution of each measurement to relative sprint speed varies among populations. Since the autocorrelation between each measurement should be considered only by this model, we defined the following equations to consider these correlations:

$$
\begin{aligned}
\mathrm{HL}_{i} & \sim \operatorname{Normal}\left(\beta_{5, k} \bullet S V L_{i}+e p_{2, k}, \sigma_{2}^{2}\right) \#(2) \\
\mathrm{HF}_{i} & \sim \operatorname{Normal}\left(\beta_{6, k} \bullet S V L_{i}+e p_{3, k}, \sigma_{3}^{2}\right) \#(3) \\
\operatorname{Tail}_{i} & \sim \operatorname{Normal}\left(\beta_{7, k} \bullet S V L_{i}+e p_{4, k}, \sigma_{4}^{2}\right) \#(4)
\end{aligned}
$$

where $\beta_{5, k}, \beta_{6, k}$, and $\beta_{7, k}$ are the coefficients of SVL, $e p_{2, k}, e p_{3, k}$, and $e p_{4, k}$ are fixed effects, and $\sigma_{2}^{2}, \sigma_{3}^{2}, \sigma_{4}^{2}$ are the variances showing individual heterogeneity.

To uncover the functional links between morphology, performance and fitness (Arnold 1983) for lizards, we estimated selection gradients and shapes of natural selection on hind leg length. We determined if lizards with longer hind legs showed higher rates of survival from predation using tail break distance as a proxy for predator escape success. We assumed individuals with longer tail break distances relative to SVL to be more capable of escaping predatory events, and therefore more likely to survive. We thus examined the survival component of fitness related to hind leg length and sprint speed by comparing tail break distance as a function of hind leg length for two snake-free islands (Miyake and Hachijo-Kojima) and two islands with snakes (Kozu and Mikura). To correct for the effect of SVL on tail break distance and hind leg length, we calculated their relative values (rTBD and rHLL, respectively) as ratios of SVL. We then applied a secondorder polynomial regression to estimate selection gradients (Equation 5), and a P-spline function (Equation 6) to visualize the shape of natural selection (Lande and Arnold 1983; Blows and Brooks 2003; Gimenez et al. 2006; Ito and Konuma 2020):

$$
\begin{gathered}
f\left(\mathrm{rTBD}_{i}\right)=\mu+\beta \cdot \mathrm{rHLL}_{i}+\frac{1}{2} \cdot \gamma \cdot \mathrm{rHLL}_{i}^{2} \#(5) \\
f\left(\mathrm{rTBD}_{i}\right)=\mu+\beta_{1} \cdot \mathrm{rHLL}_{i}+\cdots+\beta_{p} \cdot \mathrm{rHLL}_{i}^{P}+\sum_{k=1}^{K} b_{k}\left(\mathrm{rHLL}_{i}-\kappa_{k}\right)_{+}^{P} \#(6)
\end{gathered}
$$

where $\mu$ is the intercept, $\beta$ is the linear selection gradient representing directional selection, $\gamma$ is the non-linear selection gradient representing disruptive selection when $\gamma>0$ or stabilizing selection when $\gamma<0, P$ is the degree of freedom in the P-spline (which we set to 3), $\eta$ is a vector of parameters $\left(\beta_{1}, \cdots \beta_{P}, b_{1}, \cdots b_{K}\right),\left(\mathrm{rHLL}_{i}-\kappa_{k}\right)_{+}^{P}$ is either $\left(\mathrm{rHLL}_{i}-\kappa_{k}\right)^{P}$ when $\left(\mathrm{rHLL}_{i}-\kappa_{k}\right) \geq 0$ or 0 otherwise, and $\kappa_{k}$ is $k$ 's fixed knots, with $\kappa_{1}<\kappa_{2}<\cdots<\kappa_{k}$. Following Ruppert (2002) and Ito and Konuma (2020), we set the number of knots to 35 where $K=\min \left(\frac{I}{4}, 35\right)$, and used $k /(K+1)$ quantiles for all values of relative hind leg length, with $k$ between 1 and 35 . We assumed that the coefficient $b$ for $\left(\mathrm{rHLL}_{i}-\kappa_{k}\right)_{+}^{P}$ has a normal distribution with mean 0 and variance $\sigma_{b}^{2}$ (Gimenez et al. 2009). Since relative tail break distance 
was a positive continuous variable, we applied the exponential function as the link function and assumed it followed a gamma distribution.

To estimate each parameter, we used Markov Chain Monte Carlo (MCMC) simulations in a Bayesian framework. We used non-informative distributions as prior distributions for all parameters, the uniform distribution for the interval $[0,30]$ for the prior distribution of the hyper-parameters $\left(\sigma_{1}^{2}, \sigma_{2}^{2}, \ldots, \sigma_{4}^{2}\right)$, and used a normal distribution with a mean of 0 and variance of $100^{2}$ as the prior distributions for all gradients $\left(\beta_{1, k}, \beta_{2, k}, \ldots, \beta_{7, k}\right)$, fixed effects $\left(e p_{1, k}, e p_{2, k}, \ldots, e p_{4, k}\right)$, intercept $\mu$, linear selection gradient $\beta$, non-linear selection gradient $\gamma$ and the parameter $\varphi$ in the gamma distribution. We used an inverse-gamma distribution with the two parameters both at 0.001 for the prior distribution of $\sigma_{b}^{2}$. We obtained the posterior distributions for each parameter by generating three Markov chains using 2,000 MCMC simulations sampled at a rate of five times following an initial burn-in of 1,000 iterations. We standardized all measurements using z-scores in order to avoid numerical instabilities and to improve mixing each chain (Gilks \& Roberts 1996). The posterior distributions for all the parameters were summarized by $95 \%$ Bayesian confidence intervals. We used Gelman and Rubin statistics to evaluate the MCMC convergence (Gelman \& Shirley 2011) and confirmed all parameters underwent acceptable convergence. We performed MCMC simulations using PyStan (https://github.com/stan-dev/pystan; accessed 15 December 2019) in Python, and deposited the source code for the Bayesian model in $\$ \$ \$ \$ \$ \$ \$$ (e.g. GITHUB, ZENODO).

\section{RESULTS}

\section{Foraging body temperatures, snake predator presence, and recent climatic warming}

Lizards on islands with the snake predator forage at warmer body temperatures than those on islands without (Fig. 1), with respective mean foraging body temperatures of $35.4 \pm 0.1^{\circ} \mathrm{C}(n=223 ; 135$ on Kozu, 42 on Mikura, 14 on Niijima, 14 on Oshima, and 18 on Toshima) and $32.2 \pm 0.2^{\circ} \mathrm{C}(n=219 ; 85$ on Hachijo-Kojima and 134 on Miyake). After controlling for data collection period and measured air temperature in the shade with the linear mixed-effects model, we found lizard foraging body temperatures to be $2.9^{\circ} \mathrm{C}$ hotter $(p<0$. 01) on average on snake predator islands than on snake-free islands (Table S3).

We observed a warming trend across islands, with annual average daily means having increased from $16.9 \pm$ $0.2^{\circ} \mathrm{C}$ in $1981-1991$ to $18.3 \pm 0.2^{\circ} \mathrm{C}$ in $2018-2019$ (Fig. 2). We also found mean foraging body temperatures to have increased from $35.2 \pm 0.2^{\circ} \mathrm{C}$ in 1981-1991 $(n=183 ; 111$ on Kozu, 42 on Mikura, 12 on Oshima and 18 on Toshima) to $36.4 \pm 0.2^{\circ} \mathrm{C}$ in $2018-2019(n=40 ; 24$ on Kozu, 14 on Niijima and 2 on Oshima) on islands where the snake predator is present, and from $31.7 \pm 0.2^{\circ} \mathrm{C}$ in 1981-1991 ( $n=134$, all on Miyake) to $32.6 \pm 0.3^{\circ} \mathrm{C}$ in $2018-2019$ ( $n=73$, all on Hachijo-Kojima) on islands where it is absent (Fig. 2$)$. We found overall lizard foraging body temperatures in 2018-2019 to have, on average, increased by $1.3^{\circ} \mathrm{C}(\mathrm{p}<0.001)$ relative to 1981-1991 (Table S3).

\section{Thermal dependency of locomotion}

From the thermal performance curves based on racetrack sprint speeds for Kozu and Hachijo-Kojima (Fig. $3 \mathrm{~A})$ we found Kozu lizards $(n=81)$ to have maximum sprint speeds at warmer temperatures than HachijoKojima lizards $(n=85)$, with a difference in $T_{\text {opt }}$ of $1.4^{\circ} \mathrm{C}$ (Table S4). From the thermal performance curves based on open field speeds, we found Kozu lizard sprint speeds $(n=79)$ to surpass snake crawling speeds ( $n=61 ; 34$ from Kozu and 27 from Niijima) at temperatures of $33.8{ }^{\circ} \mathrm{C}$ and over (Fig. 3B). Accordingly, the mean foraging body temperature of Kozu lizards is approximately $1.6^{\circ} \mathrm{C}$ above this threshold at which Kozu lizards outpace snakes, and about $1.6^{\circ} \mathrm{C}$ below it for Hachijo-Kojima lizards. Due to the nature of the distribution of the data on Kozu lizard open field sprint speeds, however, none of the models yielded the typical unimodal shaped curve, and we could therefore not extract thermal performance parameter estimates for this particular group.

\section{Limb morphology and natural selection}

In proportion to SVL, we found Kozu lizards $(n=37)$ to have significantly longer hind legs $(0.44 \pm 0.03$, $p<0.001)$ and hind foot lengths $(0.21 \pm 0.002, p<0.001)$ than Hachijo-Kojima lizards $(0.41 \pm 0.003$ and 
$0.20 \pm 0.002$, respectively, $n=66)$. We found differences in estimated contributions of morphological traits to relative sprint speed to be especially pronounced for hind leg length and SVL (Table S5). We determined hind leg length to have a 0.82 probability of positively affecting sprint speed for Kozu lizards, and to have no effect on Hachijo-Kojima lizard sprint speed (Fig. 4A). Regarding SVL (Fig. 4D), we found a 0.91 probability of it positively affecting Hachijo-Kojima lizard sprint speed, and a 0.78 probability of it negatively affecting Kozu lizard sprint speed. We did not detect any effect of hind foot length on sprint speed for either island (Fig. 4B). Total tail length (Fig. 4C) had a 0.81 probability of positively affecting sprint speed for Kozu lizards, but had no effect for Hachijo-Kojima.

For Kozu lizards $(n=113)$, the Bayesian $95 \%$ confidence interval regarding the linear selection gradient $\beta$ did not include 0 , but the non-linear selection gradient $\gamma$ included 0 (Table S6). Accordingly, the shape of the relationship between relative hind leg length and relative tail break distance shows an upward and rising slope (Fig. 5A), implying positive directional selection on hind leg length. In contrast, for Miyake $(n$ $=78)$ and Hachijo-Kojima $(n=59)$ lizards, the Bayesian $95 \%$ confidence interval for the linear selection gradient $\beta$ included 0 (Table S6), suggesting no natural selection on hind leg length on these islands (Fig.5CD). Although for Mikura lizards $(n=38)$, the linear selection gradient confidence interval only marginally includes 0 , the shape of the relationship between hind leg length and relative tail break distance shows a rising slope (Fig. 5B) similar to that for Kozu lizards.

\section{DISCUSSION}

We demonstrate that lizard foraging body temperatures are higher in the presence of its snake predator (Fig. 1). In tandem with warmer-adapted thermal performance and predation-induced selection for longer hind legs, we show that these higher body temperatures are conducive to optimal sprint speeds at temperatures suboptimal for the predator (Fig. 3; Table S4). We also found lizard body temperatures to have increased throughout the study period, consistent with recent climatic warming over the past few decades, regardless of snake predator presence or absence (Fig. 2). This suggests a pathway through which predation in ectotherm systems acts as a pivotal factor determining prey thermal biology and thereby potentially regulating the degree of climate change vulnerability. Given the functional importance of body temperature in evasion by prey and success by predator, warming is also likely to impact ectotherm predator-prey relationships, but in unpredictable ways.

Analyses and conclusions based on thermal performance curves are often founded on many assumptions regarding their implications for fitness and resulting climate change consequences (Sinclair et al. 2016). While a significant body of work has consolidated the mechanisms through which ectotherms are vulnerable to warming impacts according to their thermal performance (Sinervo et al. 2010; Kearney 2013; Logan et al. 2013; Sinclair et al. 2016) or exposure to predation (Schmitz and Barton 2014; Sinclair et al. 2016; Laws 2017; Osmond et al. 2017), few studies have explicitly linked predation and fitness within a thermal framework. Elucidating the relationship between predation pressure and fitness, and how this affects thermal physiology, contextualizes observable differences in prey behaviour as a potentially decisive factor in climate change vulnerability (Sinervo et al. 2010; Kearney 2013). Here we show that predation-induced selection on morphology and thermal performance results in warmer prey foraging body temperatures, where further climatic warming could differentially affect these populations relative to those free from the same predation type.

In mediating tradeoffs for survival, prey species can be pushed by predation pressure to forage during hotter times of day (Tambling et al. 2015; Veldhuis et al. 2020). Yet a common expectation for lizard species is for body temperatures to be lower under higher predation pressure due to the heightened risks of behavioural thermoregulation (Huey and Slatkin 1976; Salazar et al. 2019). In contrast, we provide evidence for predation pressure by snakes to push lizard prey thermal biology towards warmer temperatures. Main predation type is also likely to be an important factor affecting prey thermal biology, seeing that $P$. latiscutatus on snakefree islands are nonetheless hunted by birds despite foraging at lower body temperatures than snake island populations. Evasion strategies by prey lizards differ depending on predator typee, where cryptic strategies are usually employed to avoid predation by birds, and running for escaping terrestrial predators (Samia et 
al. 2016). Accordingly, P. latiscutatus individuals on snake-free islands tend to carry more cryptic dorsal patterns and coloration (Brandley et al. 2014; Kuriyama et al. 2016), whereas those under snake predation are better suited physiologically and morphologically for escaping snake predation by running (Figs. 3 and 4). Broadly, prey species are often exposed to a variety of predatory strategies and densities, and understanding how these affect prey behaviours, including thermal responses, are crucial in explaining their current spatial distributions (Wirsing et al. 2010). This in turn could be important for predicting future spatial distributions under climate change as modeling tools are increasingly inclusive of interspecific interactions (Singer et al. 2016).

Body temperatures in lizard species overall tend to follow temporal environmental temperature patterns, such as within a day, or across seasons or years (Clusella-Trullas and Chown 2014; Domínguez-Guerrero et al. 2020), yet the stability or steepness of this relationship can vary depending on the number of predators or competitors present (Salazar et al. 2019). In this regard, our observations suggest body temperatures follow increases in environmental temperatures regardless of predation exposure. Yet we demonstrate that predation does drive warmer body temperatures, and with climatic warming likely to be ongoing in the near future, could thereby impose changes in lizard activity restrictions differently than for populations free from snake predation and which depend on relatively lower body temperatures (Sinervo et al. 2010; Kearney 2013). Accordingly, we provide evidence for thermal physiology to be among the life history traits through which higher rates of predation can improve prey persistence under environmental warming (Osmond et al. 2017). Conversely, prey activity windows can also be more restricted to the hottest times of day, and thus less flexible to respond to warming because of predators hunting during cooler hours (Veldhuis et al. 2020). Changes in the temporal and spatial occupancy of thermal habitats by both prey and predator, and the ways in which they compound and affect one another, thus need to be jointly considered in order to adequately estimate climate change responses (Schmitz and Barton 2014) for effective comparisons with systems free of predation.

Set within a natural laboratory system, our study provides valuable empirical evidence on how predation shapes prey behaviour, morphology and physiology, all of which are integral components of thermal adaptation (Angilletta 2009). Such responses are instrumental in mechanistically explaining prey intraspecific variability in habitat suitability according to predation type, and, ultimately, persistence under climatic warming (e.g. Landry Yuan et al. 2018). As in predation, other biotic interactions can have either negative or positive effects on environmental change vulnerability of species, as mediated by both direct and indirect pathways (Singer et al. 2016; Engelhardt et al. 2020). Thus, the implementation of holistic approaches incorporating the interplay between abiotic and biotic factors as well as life history traits is essential in breaking down the processes through which species affect one another, in order to understand biodiversity change in this era of rapid anthropogenic change.

\section{REFERENCES}

Aerts, P., Van Damme, R., Vanhooydonck, B., Zaaf, A. and A. Herrel. 2000. Lizard locomotion: how morphology meets ecology. Neth. J. Zool. 50:261-278.

Angilletta, M. J. 2006. Estimating and comparing thermal performance curves. J. Therm. Biol. 31:541-545.

Angilletta, M. J. 2009. Thermal adaptation: a theoretical and empirical synthesis . Oxford University Press.

Arnold S. J. 1983. Morphology, performance and fitness. Am. Zool.23:347-361.

Bartoń, K. 2020. Multi-Model Inference. https://cran.r-project.org/web/packages/MuMIn/index.html.

Bonine, K.E. and T. Garland Jr. 1999. Sprint performance of phrynosomatid lizards, measured on a highspeed treadmill, correlates with hindlimb length. J. Zool. 248:255-265.

Blows, M. W. and R. Brooks. 2003. Measuring Nonlinear Selection.Am. Nat. 162: 815-20.

Brandley, M. C., Kuriyama, T. and M. Hasegawa. 2014. Snake and bird predation drive the repeated convergent evolution of correlated life history traits and phenotype in the Izu Island scincid lizard (Plestiodon 
latiscutatus ). PLOS ONE 9: e922

Burnham, K. P. and D. R. Anderson. 2002. Model selection and multimodel inference: a practical information-theoretic approach. Springer Science and Business Media.

Chai, P. and R. B. Srygley. 1990. Predation and flight, morphology, and temperature of neotropical rainforest butterflies. Am. Nat.135:748-765.

Clusella-Trullas, S. and S. L. Chown. 2014. Lizard thermal trait variation at multiple scales: a review. J. Comp. Physiol. B.184:5-21.

Cooper Jr, W. E. 2000. Effect of temperature on escape behaviour by an ectothermic vertebrate, the keeled earless lizard (Holbrookia propinqua). Behaviour 137:1299-1315.

De Boeck, P., Bakker, M., Zwitser, R., Nivard, M., Hofman, A., Tuerlinckx, F., et al. 2011. The estimation of item response models with the lmer function from the lme4 package in R. J. Stat. Softw. 39:1-28.

Dell, A. I., Pawar, S. and V. M. Savage. 2014. Temperature dependence of trophic interactions are driven by asymmetry of species responses and foraging strategy. J. Anim. Ecol. 83:70-84.

Domínguez-Guerrero, S. F., Bodensteiner, B. L., Pardo-Ramirez, A., Aguillon-Gutierrez, D. R., Mendez-de la Cruz, F. R. and M. M. Munoz. 2020. Thermal physiology responds to interannual temperature shifts in a montane horned lizard, Phrynosoma orbiculare . J. Exp. Zool. Part A. https://doi.org/10.1002/jez.2403

Engelhardt, E. K., Neuschulz, E. L. and C. Hof. 2020. Ignoring biotic interactions overestimates climate change effects: The potential response of the spotted nutcracker to changes in climate and resource plants. J. Biogeogr. 47:143-154.

Figueira, W. F., Curley, B. and D. J. Booth. 2019. Can temperature-dependent predation rates regulate range expansion potential of tropical vagrant fishes? Mar. Biol. 166:73.

Gelman, A. and K. Shirley. 2011. Inference from simulations and monitoring convergence. In Handbook of Markov Chain Monte Carlo, ed. Brooks, S., Gelman, A., Jones, G. L. and X. L. Meng. Chapman and Hall/CRC, London/Boca Raton, 163-174.

Gilks, W. R. and G. O. Roberts. 1996. Strategies for improving MCMC. InMarkov chain Monte Carlo in practice, ed. Gilks, W. R., Richardson, S. and D. J. Spiegelhalter. Chapman and Hall, New York, 89-114.

Gimenez, O., Crainiceanu, C., Barbraud, C., Jenouvrier, S. and B. J. T. Morgan. 2006. Semiparametric Regression in Capture-Recapture Modeling.Biometrics 62: 691-98.

Gimenez, O., Gregoire, A., and T. Lenormand. 2009. Estimating and Visualizing Fitness Surfaces Using Mark-Recapture Data. Evolution63: 3097-3105.

Grigaltchik, V. S., Ward, A. J. and F. Seebacher. 2012. Thermal acclimation of interactions: differential responses to temperature change alter predator-prey relationship. Proc. R. Soc. B.279:4058-4064.

Hasegawa, M., and H. Moriguchi. 1989. Geographic variation in food habits, body size and life history traits of the snakes on the Izu Islands. In Current Herpetology in East Asia, ed. Matui, M., Hikida, T. and R. C. Goris. The Herpetological Society of Japan, Kyoto, 414-432.

Hasegawa, M. 1990. The thrush Turdus celaenops, as an avian predator of juvenile Eumeces okadae on Miyake-jima, Izu Islands.Jpn. J. Herpetol. 13:65-69.

Hasegawa, M. 1994. Insular radiation in life history of the lizardEumeces okadae in the Izu Islands, Japan. Copeia3:732-747.

Hasegawa, M., Asada, M., Taniguchi, K. and H. Kurono. 1996. Distribution and status of gray-faced buzzard eagle Butastur indicus on the northern Izu Islands, Japan. Jpn. J. Ornithol. 45:83-89. 
Hasegawa, M. 1999. Impacts of the introduced weasel on the insular food webs. In Tropical Island Herpetofauna: Origin, Current Diversity, and Conservation, ed. H. Ota. Elsevier, 129-154.

Hasegawa, M. 2003. Ecological diversification of insular terrestrial reptiles: a review of the studies on the lizard and snakes of the Izu Islands. Glob. Environ. Res. 7:59-68.

Hertz, P. E., Huey, R. B. and E. Nevo. 1983. Homage to Santa Anita: thermal sensibility of sprint speed in agamid lizards. Evolution37:1075-1084.

Huey, R. B. and M. Slatkin. 1976. Cost and benefits of lizard thermoregulation. Q. Rev. Biol. 51:363-384.

Ito, S., and J. Konuma. 2020. Disruptive selection of shell colour in land snails: a mark-recapture study of Euhadra peliomphala simodae. Biol. J. Linn. Soc. 129: 323-33.

Kaneoka, I., Isshiki, N. and S. Zashu. 1970. K-Ar ages of the Izu-Bonin islands. Geochem. J. 4:53-60.

Kearney, M. R. 2013. Activity restriction and the mechanistic basis for extinctions under climate warming. Ecol. Lett. 16:1470-1479.

Kuriyama, T., Brandley, M. C., Katayama, A., Mori, A., Honda, M. and M. Hasegawa. 2011. A timecalibrated phylogenetic approach to assessing the phylogeography, colonization history and phenotypic evolution of snakes in the Japanese Izu Islands. J. Biogeogr. 38:259-271.

Kuriyama, T., Morimoto, G., Miyaji, K. and M. Hasegawa. 2016. Cellular basis of anti-predator adaptation in a lizard with autotomizable blue tail against specific predators with different colour vision. J. Zool. 300:89-98.

Lande, R., and S. J. Arnold. 1983. The measurement of selection on correlated characters. Evolution 37: $1210-26$.

Landry Yuan, F., Freedman, A. H., Chirio, L., LeBreton, M. and T. C. Bonebrake. 2018. Ecophysiological variation across a forest-ecotone gradient produces divergent climate change vulnerability within species.Ecography 41:1627-1637.

Laws, A. N. 2017. Climate change effects on predator-prey interactions.Curr. Opin. Insect Sci. 23:28-34.

Lleonart, J., Salat, J. and G. J. Torres. 2000. Removing allometric effects of body size in morphological analysis. J. Theor. Biol.205:85-93.

Logan, M. L., Huynh, R. K., Precious, R. A. and R. G. Calsbeek. 2013. The impact of climate change measured at relevant spatial scales: new hope for tropical lizards. Glob. Change Biol. 19:3093-3102.

Ohlund, G., Hedstrom, P., Norman, S., Hein, C. L. and G. Englund. 2015. Temperature dependence of predation depends on the relative performance of predators and prey. Proc. R. Soc. B. 282:20142254. http://dx.doi.org/10.1098/rspb.2014.2254.

Osmond, M. M., Otto, S. P. and C. A. Klausmeier. 2017. When predators help prey adapt and persist in a changing environment. Am. Nat.190:83-98.

R Core Team 2019. R: A language and environment for statistical computing. R Foundation for Statistical Computing, Vienna, Austria. Available at: https://www.R-project.org/. (last accessed 15 December 2019)

Ruppert, D. 2002. Selecting the number of knots for penalized splines.J. Comput. Graph. Stat. 11: 735-57.

Salazar, J. C., del Rosario Castaneda, M., Londono, G. A., Bodensteiner, B. L. and M. M. Munoz. 2019. Physiological evolution during adaptive radiation: A test of the island effect in Anolis lizards.Evolution 73:1241-1252.

Samia, D. S., Blumstein, D. T., Stankowich, T. and W. E. Cooper Jr. 2016. Fifty years of chasing lizards: new insights advance optimal escape theory. Biol. Rev. 91:349-366. 
Schmitz, O. J. and B. T. Barton. 2014. Climate change effects on behavioral and physiological ecology of predator-prey interactions: implications for conservation biological control. Biol. Control75:87-96.

Sinclair, B. J., Marshall, K. E., Sewell, M. A., Levesque, D. L., Willett, C. S., Slotsbo, S., Dong, Y., Harley, C. D., Marshall, D. J., Helmuth, B. S. and R. B. Huey. 2016. Can we predict ectotherm responses to climate change using thermal performance curves and body temperatures? Ecology Letters 19:1372-1385.

Sinervo, B., Mendez-De-La-Cruz, F., Miles, D. B., Heulin, B., Bastiaans, E., Villagran-Santa Cruz, M., et al. 2010. Erosion of lizard diversity by climate change and altered thermal niches. Science328:894-899.

Singer, A., Johst, K., Banitz, T., Fowler, M. S., Groeneveld, J., Gutierrez, A. G., et al. 2016. Community dynamics under environmental change: How can next generation mechanistic models improve projections of species distributions? Ecol. Model. 326:63-74.

Snell, H. L., Jennings, R. D., Snell, H. M. and S. Harcourt. 1988. Intrapopulation variation in predatoravoidance performance of Galapagos lava lizards: the interaction of sexual and natural selection.Evol. Ecol. 2:353-369.

Tambling, C. J., Minnie, L., Meyer, J., Freeman, E. W., Santymire, R. M., Adendorff, J., et al.. 2015. Temporal shifts in activity of prey following large predator reintroductions. Behav. Ecol. Sociobiol.69:11531161 .

Veldhuis, M. P., Hofmeester, T. R., Balme, G., Druce, D. J., Pitman, R. T. and J. P. G. M. Cromsigt . 2020. Predation risk constrains herbivores' adaptive capacity to warming. Nat. Ecol. Evol.https://doi.org/10.1038/s41559-020-1218-2

Whittaker, R. J., Fernandez-Palacios, J. M., Matthews, T. J., Borregaard, M. K. and K. A. Triantis. 2017. Island biogeography: Taking the long view of nature's laboratories. Science 357:p.eaam8326.

Wirsing, A. J., Cameron, K. E. and M. R. Heithaus. 2010. Spatial responses to predators vary with prey escape mode. Anim. Behav.79:531-537.

Zipkin, E. F., DiRenzo, G. V., Ray, J. M., Rossman, S. and K. R. Lips. 2020. Tropical snake diversity collapses after widespread amphibian loss. Science 367:814-816.

\section{ACKNOWLEDGEMENTS}

This work was approved by the University of Hong Kong's Committee of the Use of Live Animals in Teaching \& Research (4599-18), and financially supported with funding awarded to M. Hasegawa (19H03307, 15H04426) by the Japan Society for the Promotion of Science (JSPS). Fieldwork on Kozu and Niijima was approved by local village governments in accordance with their ordinances governing plant and animal protection, while no specific permission is required for fieldwork on other Izu Islands. We thank Yoji Kigasawa for allowing us to use his lizard body temperature data taken on Miyake island in 1983. We also thank Dr. Yosuke Kojima and Miki Hirose for their invaluable help in the field, as well as Dr. T.Y. Hui for reviewing this manuscript and providing valuable comments. MH expresses sincere thanks to the late Shouichi Sengoku and late Kazuyoshi Miyashita for their mentorship during the early phase of fieldwork from the late 1970's to late 1980 's. 


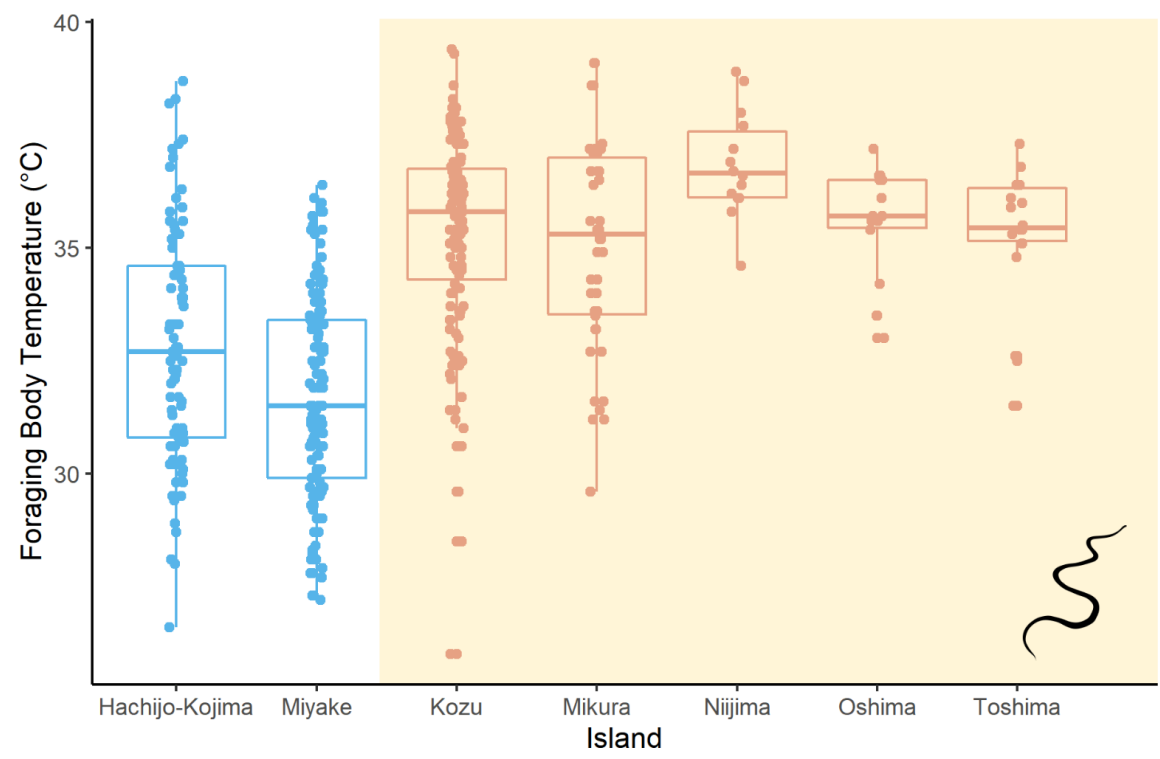

Figure 1. Boxplots depicting foraging body temperatures for P. latiscutatus on islands where E. quadrivirgata is present (orange with shaded area), and on islands where E. quadrivirgatais absent (blue with unshaded area).
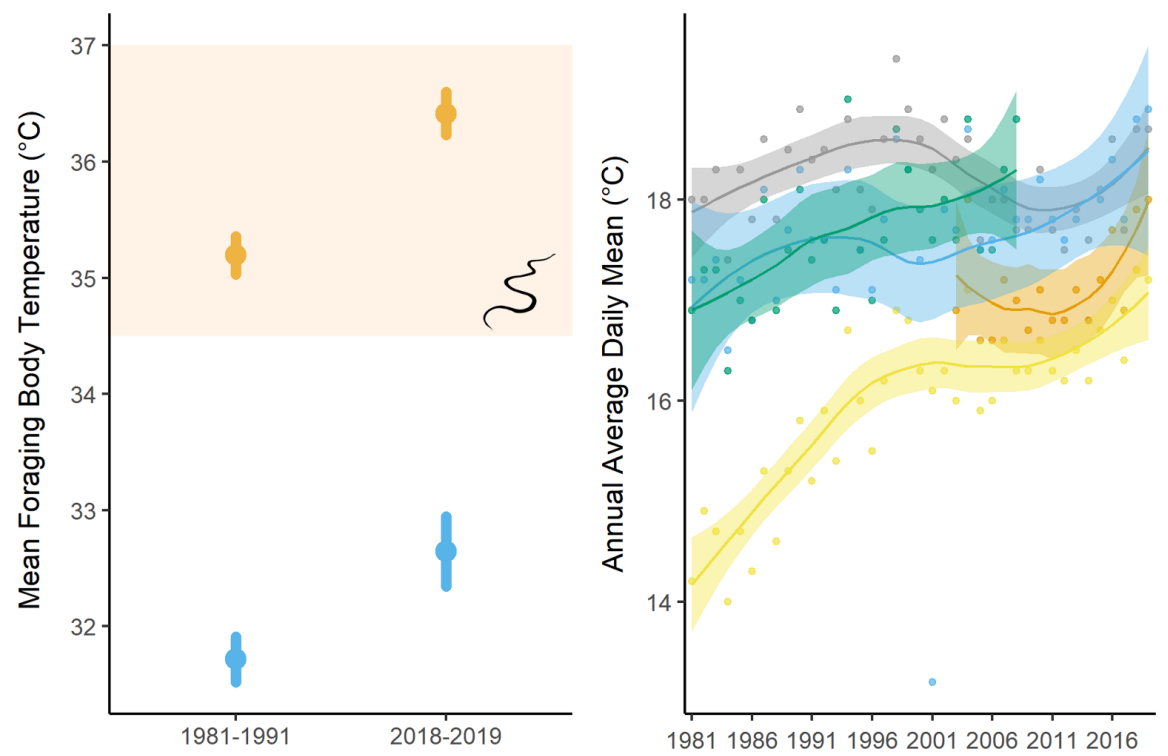

Figure 2. Shown in the left panel are the increases in mean foraging body temperature $( \pm \mathrm{SE})$, from the earliest (1981-1991) to the latest data collection periods (2018-2019), for P. latiscutatus on islands where E. quadrivirgata is present (orange with shaded area), and on islands where E. quadrivirgata is absent (blue with unshaded area). Shown in the right panel as plotted points and associatedloess curves with $95 \%$ confidence intervals are the annually averaged daily mean temperatures for the years between 1981 and 2019 for which data were available for Hachijojima (grey), Kozu (orange), Miyake (blue), Niijima (green) and Oshima (yellow). 

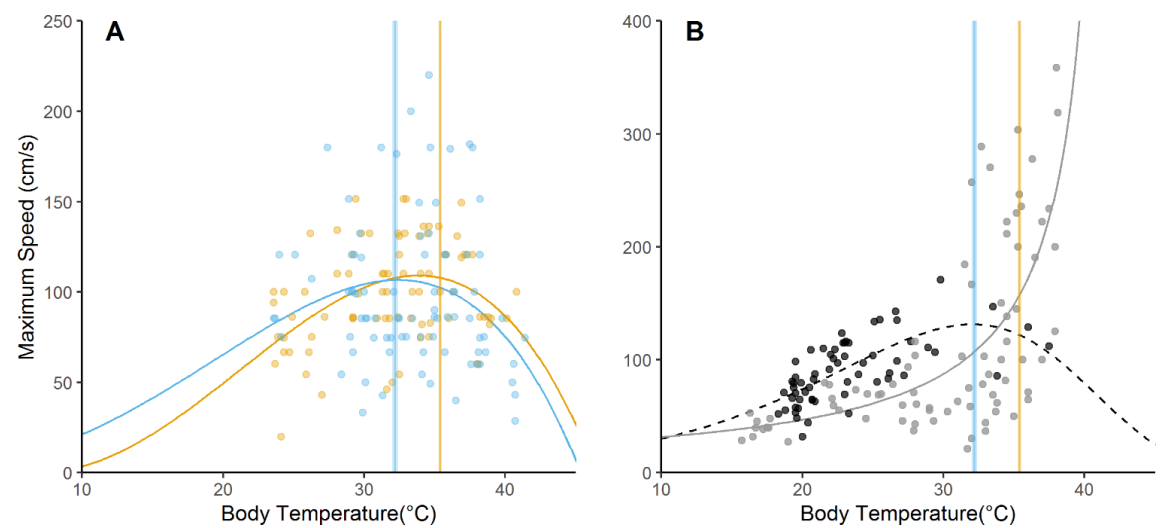

Figure 3. Thermal performance curves estimated from the non-linear models applied to the raw maximum locomotory speed data for P. latiscutatus and E. quadrivirgata. Curves in panel A are based on racetrack sprint speeds of P. latiscutatus individuals from Hachijo-Kojima (blue solid line), where E. quadrivirgata is absent, and Kozu (orange solid line), where it is present. Panel B shows the curves based on P. latiscutatus open field sprint speeds on Kozu (solid grey line) and E. quadrivirgata maximum crawling speeds on Niijima and Kozu (black dotted line). The plotted points represent individual speed measurements for HachijoKojima (blue) and Kozu (orange) P. latiscutatus in panel A, as well as for E. quadrivirgata (black) and open field $P$. latiscutatus (grey) in panel B. The vertical bands in both panels represent foraging body temperatures for P. latiscutatus measured on Hachijo-Kojima (blue) and Kozu (orange) islands, where the middle line is the mean, and the shaded range the standard error. The descending portion of theP. latiscutatus open field sprint speed curve (solid grey line) in panel B was removed for visual clarity.
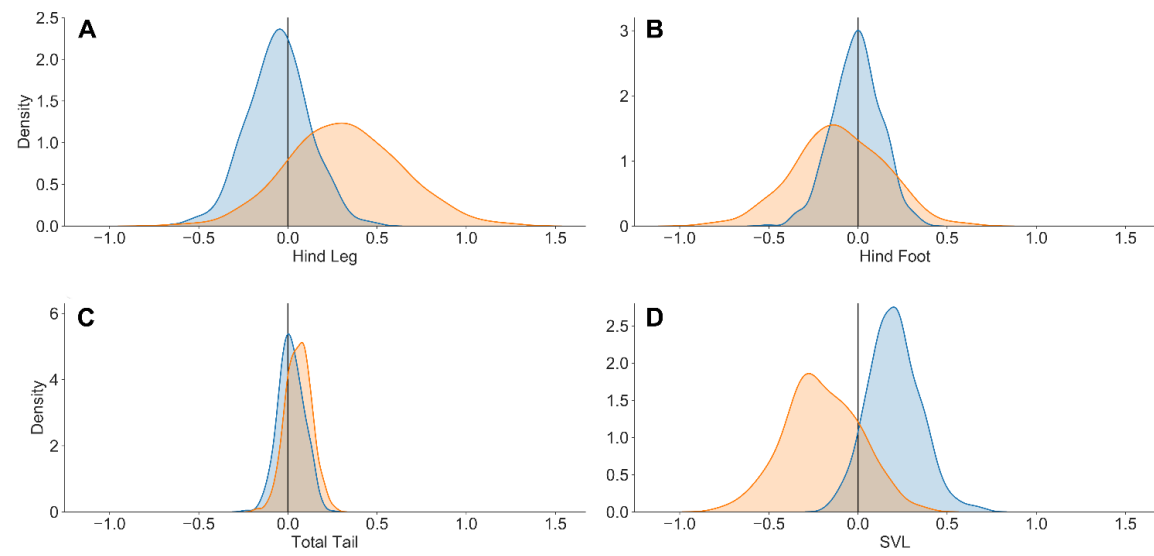

Figure 4. Posterior density plots estimating the contributions from each morphological measurement to relative sprint speed for $P$. latiscutatus individuals from Hachijo-Kojima (blue), where E. quadrivirgata is absent, and Kozu (orange), where it is present. Here, A) represents hind leg length, B), hind foot length, C), total tail length, and D), SVL. 

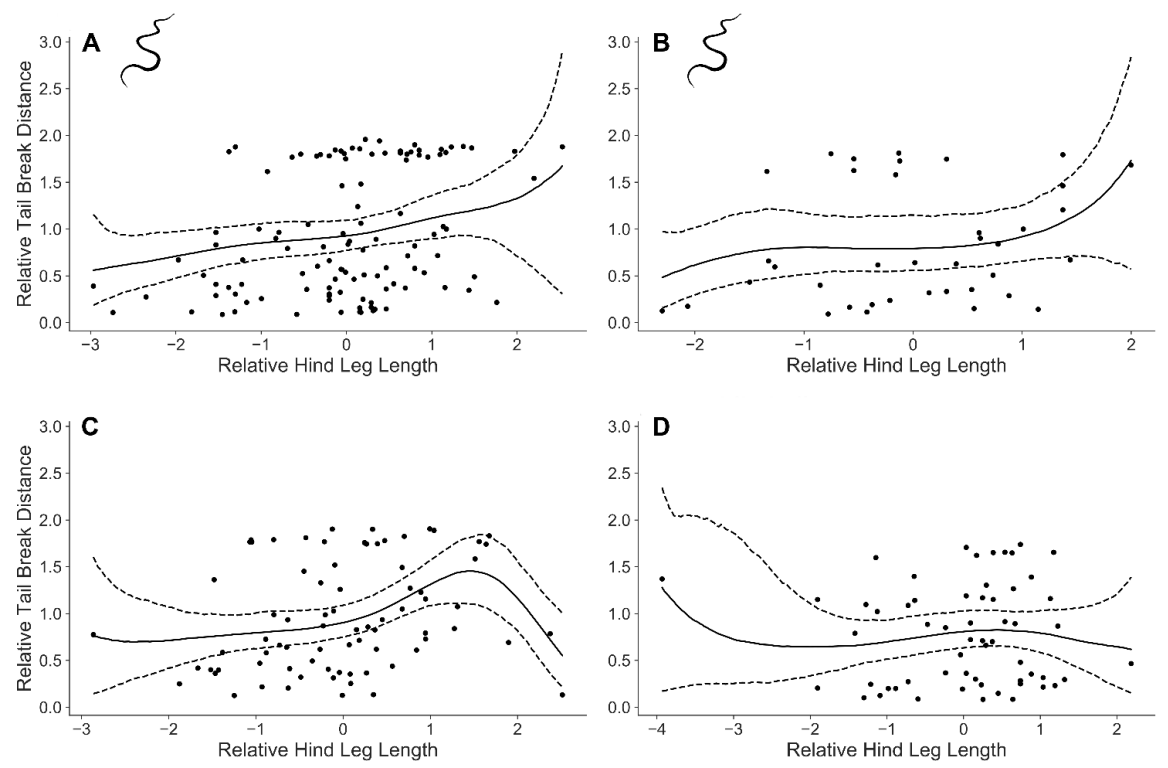

Figure 5. The relationship between relative tail break distance and relative hind leg length for $P$. latiscutatus from A) Kozu and B) Mikura, where E. quadrivirgata is present, as well as from C) Miyake and D) HachijoKojima, where E. quadrivirgata is absent. This shape represents the natural selection worked on relative hind leg. The solid and dashed lines indicate the median and the $95 \%$ Bayesian confidence intervals, respectively, in the posterior distribution obtained from Markov chain Monte Carlo simulations. Each point represents individual measurement values.

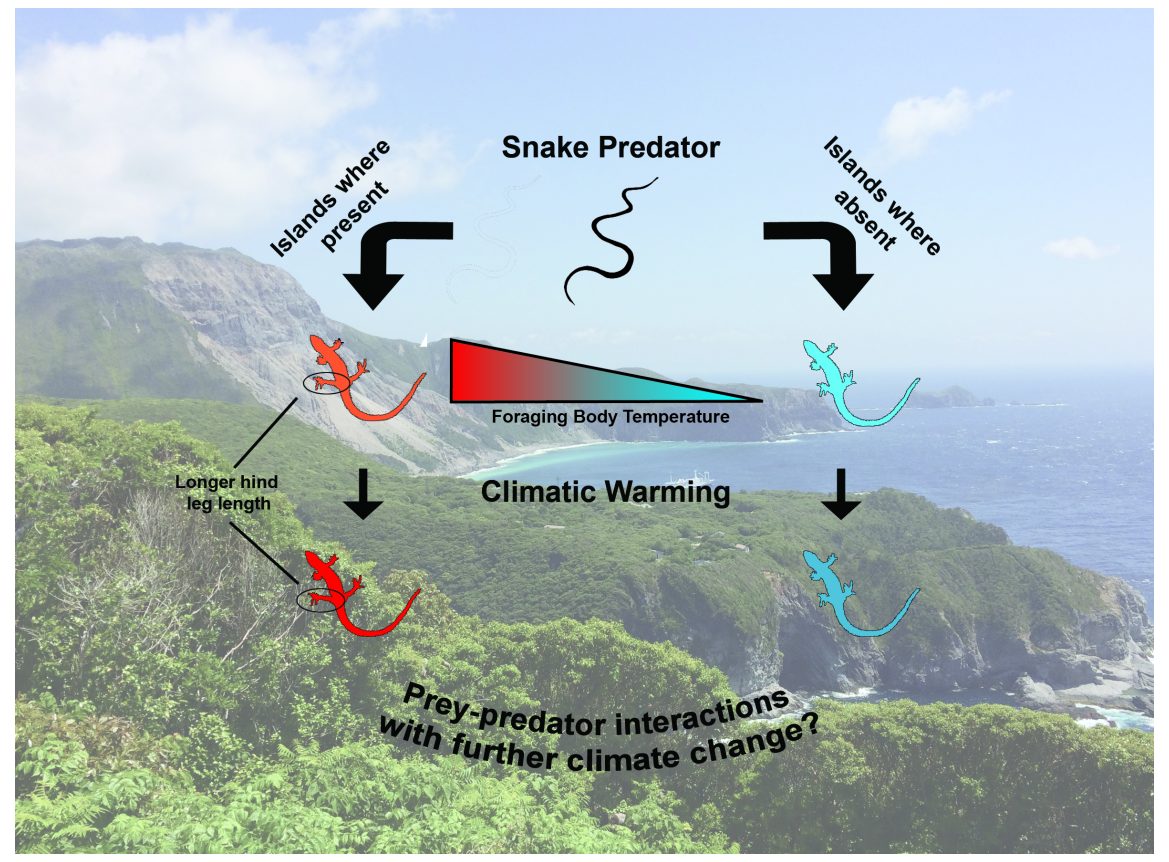

